\title{
The Bisketene Radical Cation and Its Formation by Oxidative Ring- Opening of Cyclobutenedione
}

\author{
Krzysztof Piech and Thomas Bally* \\ Department of Chemistry, University of Fribourg, CH-1700 Fribourg, Switzerland
}

\author{
Annette D. Allen and Thomas T. Tidwell \\ Department of Chemistry, University of Toronto, Ontario, Canada M5S 3H6
}

\begin{abstract}
Parent cyclobutenedione 1 was photolyzed and ionized in an Ar matrix at 10K. The bisketene 2 that results in both cases (in the form of its radical cation after ionization) was characterized by its IR spectrum and by high-level quantum chemical calculations. Experiment and theory show that the neutral bisketene has only a single conformation where the two ketene moieties are nearly perpendicular, whereas the radical cation is present in two stable planar conformations. The mechanism of the ring-opening reaction, process that involves an avoided crossing of states.

\section{INTRODUCTION}

Vicinal bisketenes are very reactive compounds ${ }^{1,2}$ and are also useful synthons that have been used to prepare mixed and symmetrical succinate esters, ${ }^{\text {1f }}$ butenolides, ${ }^{\text {If }}$ symmetrical and mixed succinamides, ${ }^{2 \mathrm{~g}}$ succinimides, $^{2 \mathrm{~g}}$ ester amides, ${ }^{2 \mathrm{~g}}$ ortho esters, $^{2 \mathrm{e}}$ and other species, including aziridines (eq 1$)^{1 \mathrm{~d}}$
\end{abstract}

both in the neutral and in the radical cation, is discussed on the basis of calculations. In the latter case it is a nonsynchronous

$$
\text { (R) }
$$

Bisketenes are more reactive than monoketenes resulting from the first reaction, and so selective reactions in which the two ketenyl groups are successively reacted first with one reagent and then with another are possible (eq 2). ${ }^{2 g}$

Thus, bisketenes react with aldehydes to give isolable $\beta-$ lactones which on pyrolysis lose $\mathrm{CO}_{2}$ to give vinylketenes (eq $3),{ }^{1 \mathrm{~h}}$ and reactions with diazoalkanes give cyclopentenediones and lactones (eq 4). ${ }^{1 \mathrm{i}}$ Bisketenes also undergo $[2+2]$ cycloaddition with cyclopentadiene forming spirobutenolides and ketenylcyclobutanones ${ }^{2 \mathrm{i}, \mathrm{j}}$ and $[4+2]$ cycloadditions with alkynes forming $p$-benzoquinones, together with spiro products of $[2+1]$ cycloaddition with bicyclization (eq 5). ${ }^{2 \mathrm{~h}}$ Reactions

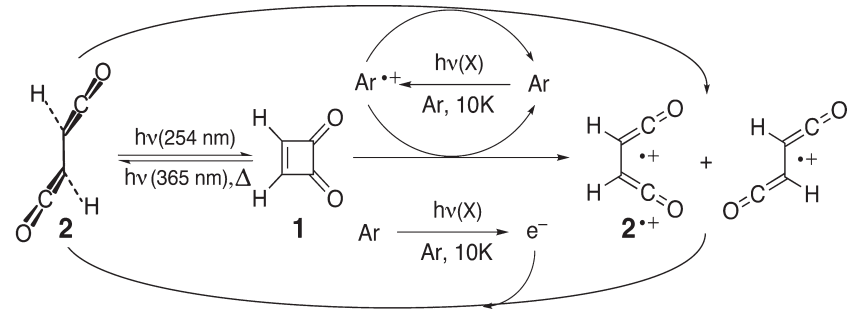




$$
\begin{aligned}
& \overbrace{\mathrm{O}}^{\mathrm{O}}=\mathrm{C}_{\mathrm{C}=\mathrm{O}}^{\mathrm{h}} \\
& 12
\end{aligned}
$$

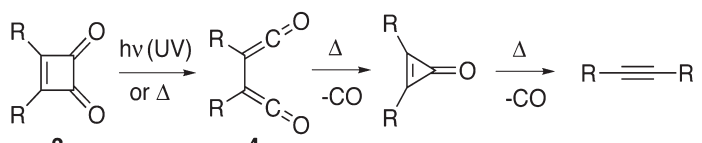

$$
\begin{aligned}
& 3 \\
& { }^{\mathrm{N}_{2}} \mathrm{O} \text { hv(UV) } \mathrm{H}_{Y}=\mathrm{O}
\end{aligned}
$$

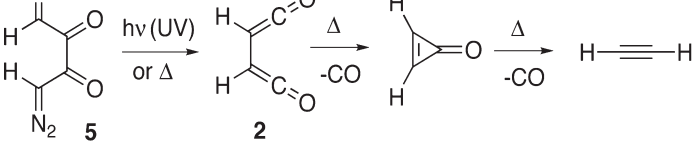

The parent bisketene $\mathbf{2}$ has also been generated and observed upon matrix photolysis in Ar at $10 \mathrm{~K}$ of the bis(diazo ketone) 5 and underwent decarbonylation to give cyclopropenone and then acetylene upon prolonged photolysis (eq 8). ${ }^{2 a}$ Recently, dimethoxybisketene $(4, \mathrm{R}=\mathrm{OMe})$ was similarly generated in an Ar matrix from the dimethyl ester of squaric acid (3, R = $\mathrm{OMe}){ }^{2 \mathrm{~b}}$ Photolysis of benzocyclobutenedione gave the bisketene 6 characterized in Ar at $11 \mathrm{~K}$ (eq 9), ${ }^{2 \mathrm{c}}$ and a variety of other unstable vicinal bisketenes have been generated in solution and characterized by time-resolved UV or IR spectroscopy. $^{2 \mathrm{~d}}$

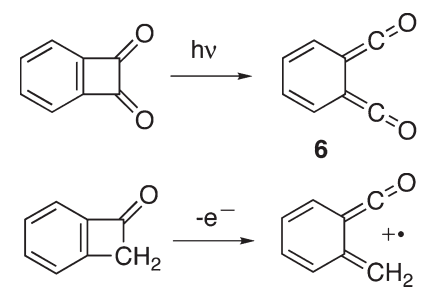

We had previously observed that upon ionization benzocyclobutenone undergoes spontaneous ring-opening to yield the radical cation of the quinoketene, 6-methylene-2,4-cyclohexadiene-1-ylidenemethanone (eq 10). ${ }^{3}$ We therefore wanted to see whether the ring-opening of cyclobutenedione could perhaps also be induced by ionization, thus opening a new avenue to the generation of bisketenes, e.g., by single-electron transfer.

Thus, we subjected parent cyclobutenedione 1 to photolysis in Ar matrices to characterize the resulting parent bisketene, 2, and then to X-irradiation which partially converts neutral compounds that are contained in Ar matrices to their radical cations or to secondary products. ${ }^{4}$ This paper describes the results of these experiments and the accompanying quantum chemical calculations, which help to assign the spectra and understand the chemistry that we have observed.

\section{RESULTS AND DISCUSSION}

IR Spectra. Figure 1 sums up the results of the different experiments we did with cyclobutenedione. Obviously, on 254 nm photolysis, $\mathbf{1}$ is converted to $\mathbf{2}$, as evidenced by the three strong IR bands that arise at 2114, 2127, and $2140 \mathrm{~cm}^{-1}$. According to our (and previous) ${ }^{1 a}$ calculations, the $C_{2}$ equilibrium conformation of 2 is twisted by nearly $90^{\circ}$ and it shows a symmetric and an antisymmetric combination of the strongly IR-active ketene stretching vibrations. In addition, we found a shallow secondary minimum which corresponds to a planar $C_{2 h}$ (trans) structure, with only a single IR band in this spectral region (the antisymmetric combination of ketene stretches is not IR active in this case), ca. $1 \mathrm{kcal} / \mathrm{mol}$ above the $\mathrm{C}_{2}$ minimum (see Figure 2).

However, the activation enthalpy for the decay of the metastable trans- $C_{2 \mathrm{~h}}$ to the twisted $C_{2}$ equilibrium structure is vanishingly small, if it exists at all (high-level calculations, as they are used in the $\mathrm{G} 4$ procedure, place the transition state found by DFT calculations ca. $1 \mathrm{kcal} / \mathrm{mol}$ below the $C_{2 h}$ minimum; see Table $S 1$ in the Supporting Information). Thus, the $C_{2 h}$ structure of $\mathbf{2}$ is unlikely to persist, and although the presence of both conformers of $\mathbf{2}$ in the matrix would nicely explain the observation of three bands, we favor an interpretation of the three bands as being due to a site splitting, as it is invariably observed in matrix IR spectra of compounds with cumulated double bonds. As the last of these bands is broader than the other two, and it peaks at $2140 \mathrm{~cm}^{-1}$, i.e., almost exactly where $\mathrm{CO}$ absorbs in Ar matrices, we also cannot exclude that some $\mathrm{CO}$ is expelled in the course of the photolysis, although we will show below that this is rather unlikely.

Why are bisketenes 2 twisted almost $90^{\circ}$ ? Probably for two reasons: first, the HOMO of $\mathbf{2}$ is as $\pi$-antibonding along the central $\mathrm{C}-\mathrm{C}$ bond as the subjacent $\pi$-MO is bonding, so the $\pi$ bond order along this bond is almost zero, in contrast to butadiene where the lowest $\pi$-MO is much more bonding than the HOMO is antibonding, which results in a positive $\pi$-bond order. ${ }^{5}$ Second, the perpendicular geometry minimizes the unfavorable dipole interactions between the two $\mathrm{C}=\mathrm{O}$ groups which prevail in the planar structures, in particular in the $C_{2 v}$ one.

If, instead of exposing an Ar matrix containing 1 to UV light, it is subjected to $90 \mathrm{~min}$ of X-irradiation, the green difference spectrum (b) in Figure 1 is obtained (for an explanation of the events that occur on X-irradiation of Ar matrices, see Methods).

At first sight this spectrum looks very similar to spectrum (a); i.e., the two peaks of $\mathbf{1}$ decrease and the three of $\mathbf{2}$ increase, albeit with different relative intensities than after photolysis of 1. However, closer inspection reveals some new bands between 2150 and $2300 \mathrm{~cm}^{-1}$ that had not arisen upon photolysis (see the enlargement in Figure 1), some of which we will assign to the radical cation of $\mathbf{2}$.

It is known that, after ca. 90 min of X-irradiation, the amount of charged species that are formed in an Ar matrix levels off because they are then reneutralized by the eletrons that roam through the matrix at the same rate as they are formed by scavenging the holes. If the formed radical cations do not undergo any chemistry, additional $\mathrm{X}$-irradiation results in no spectral changes. However, if the incipient radical cations rearrange, as it is the case here, then the rearranged cations get reneutralized and the formation of rearranged neutral (in our case 2) can continue, in principle indefinitely.

The results of further X-irradiation (orange difference spectrum (c)) are shown to demonstrate that, under these conditions, no additional $2^{\bullet+}$ is formed (the three bands labeled " $c$ " do not increase) because it is continually reneutralized to give more 2 , as evidenced by the increase of its characteristic triplet of bands (see Methods). In addition, this experiment shows that the two small peaks labeled " $n$ " also continue to increase, which indicates that they cannot belong to charged species but must be due to a neutral byproduct that may perhaps form upon loss of CO (unfortunately, CO escapes our detection because its comparatively weak IR absorption lies within the strong triplet of peaks of 2 , and spectrum (c) does 


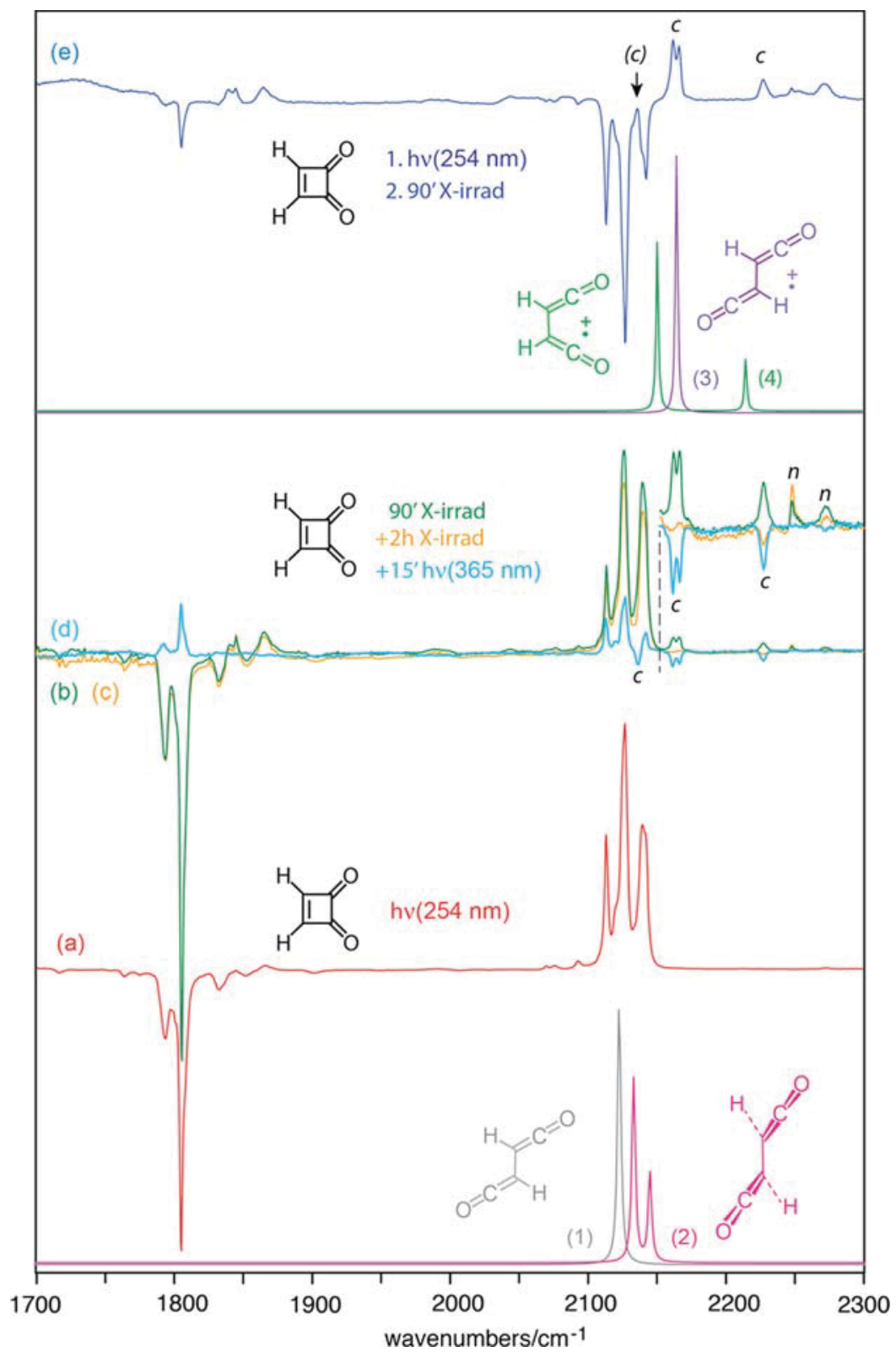

Figure 1. Difference spectra for (a) photolysis of $\mathbf{1}$ at $254 \mathrm{~nm}$, (b) $90 \mathrm{~min}$. X-irradiation of $\mathbf{1}$, (c) additional $2 \mathrm{~h}$ of X-irradiation of $\mathbf{1}$, (d) subsequent photolysis at $>515 \mathrm{~nm}$, and (e) X-irradation of a sample of $\mathbf{1}$ that had previously been photolysed to give 2 . Bands in spectra c-e labeled " $c$ " are due to cations, and those labeled " $n$ " are neutral species (see the discussion in text). The spectra of the different species (traces 1-4) are from B3LYP/6$31 \mathrm{G}^{*}$ calculations, scaled by 0.968 for the neutrals, 0.960 for the cations.

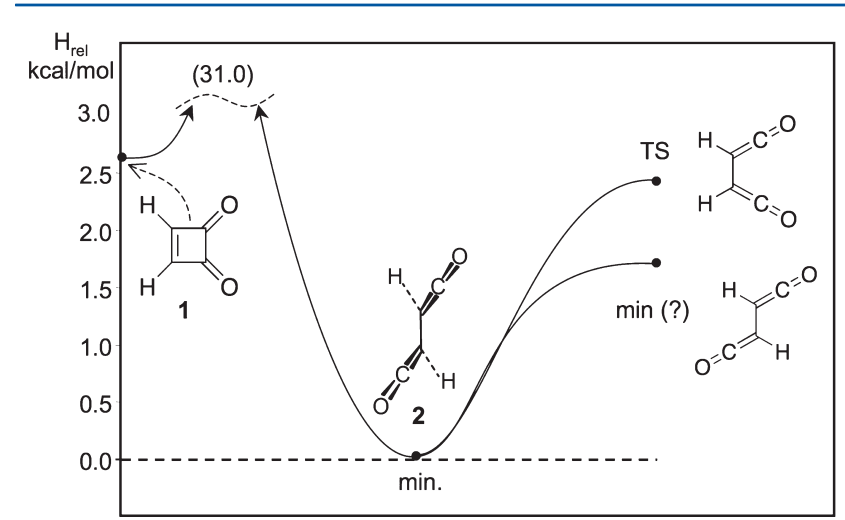

Figure 2. G4 potential surface for the conversion of 1 to 2 and the interconversion of different conformers of 2 . not reveal any evidence for the characteristic pair of intense bands at 1780 and $1880 \mathrm{~cm}^{-1}$ of cyclopropenone. ${ }^{6,7}$

As X-irradiation of an Ar matrix containing 1 resulted also in the increase of some weak bands in the near-UV part of the spectrum (which otherwise looks too nondescript to serve as a valid source of chemical information), we decided to expose the ionized sample to $365 \mathrm{~nm}$ irradiation. This did indeed result in a decrease of the bands labeled " $c$ " in Figure 1d, and the rise of the triplet of peaks of $\mathbf{2}$, which suggests that this photolysis led to some reneutralization of $2^{\bullet+}$. At the same time, the peaks of 1 also grow, albeit only to a small extent, indicating that a small amount of recyclization takes place. The most interesting aspect of that experiment is, however, that a band at ca. $2130 \mathrm{~cm}^{-1}$ decreases, in concert with the other bands labeled " $c$ ", which suggests that this band belongs to the same species. 


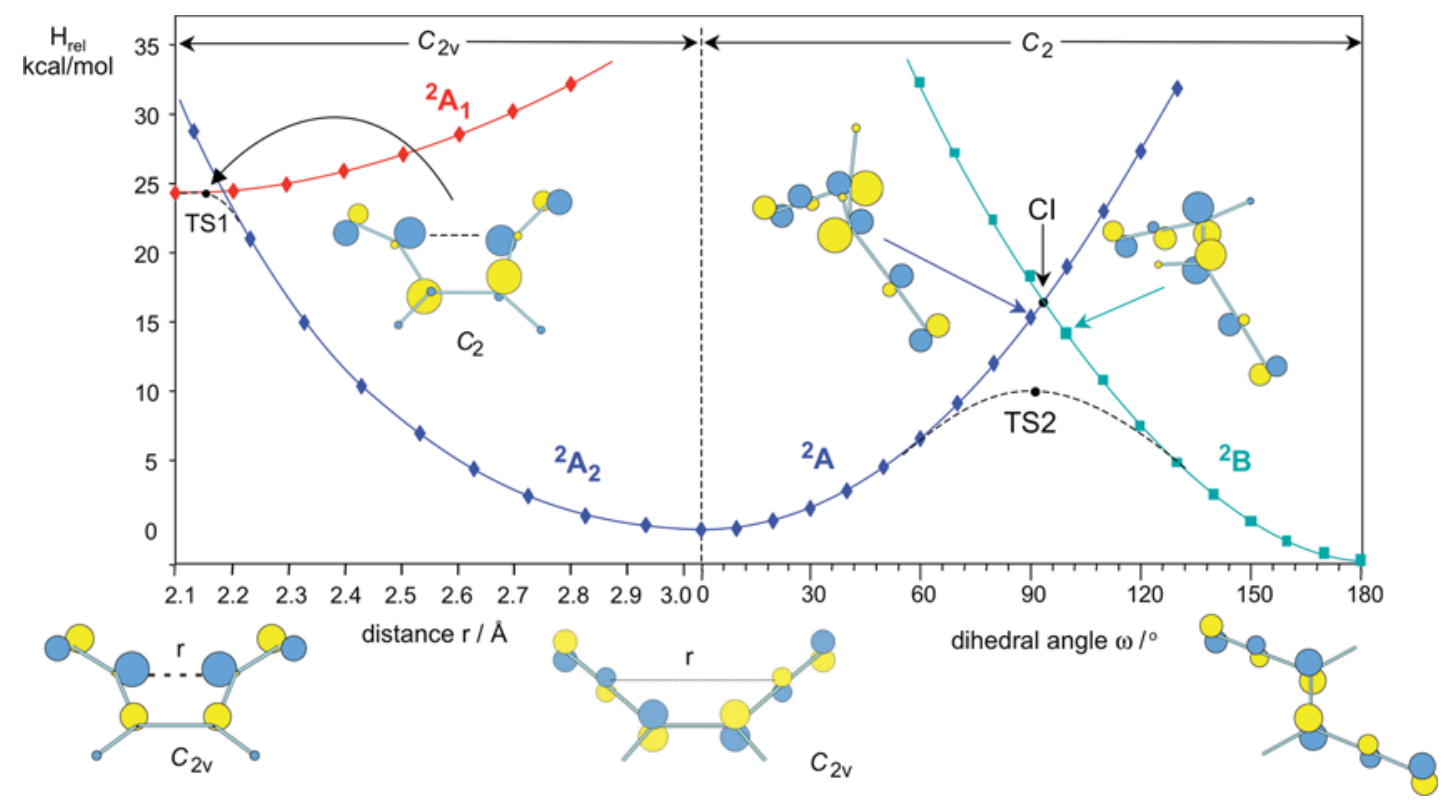

Figure 3. B3LYP/6-31G* potential surfaces for the interconversion of $\mathbf{1}^{\bullet+}$ to $c$ is- $2^{\bullet+}$ and for the interconversion of the latter to trans-2 $2^{\bullet+}$. The orbital plots represent the singly occupied $\mathrm{MO}$ (SOMO) in each state.

Finally, we preformed 2 by photolysis of 1 before Xirradiation, which gave rise to the difference spectrum (e) at the top of Figure 1. This time the bands of $\mathbf{2}$ decrease (because $\mathbf{2}$ is ionized), and the same bands above $2150 \mathrm{~cm}^{-1}$ increase as in the experiment where $\mathbf{1}$ had been ionized (green spectrum (b)). The "c" band at $2130 \mathrm{~cm}^{-1}$ does not point up as much as it should because its increase is overcompensated by the decrease of the $2140 \mathrm{~cm}^{-1}$ band of 2 , but the mere fact that this latter band is much smaller in the difference spectrum (d) than in the others indicates that some other absorption must be increasing in this region.

The above-described observations lead us to conclude that we see three IR-absorptions of $2^{\bullet+}$, at 2140, 2162/2166 (double peak) and $2227 \mathrm{~cm}^{-1}$. Quantum chemical calculations of $2^{\bullet+}$ reveal that it exists in two stable conformations, cis $\left(C_{2 v}\right)$ and trans $\left(C_{2 h}\right)$, the first of which shows a pair of widely spaced ketene stretching vibrations while the second shows only one IR-active band in this region, due to its symmetry (see simulated spectra 3 and 4, respectively, in Figure 1). These results allow us to propose that the double peak at ca. 2164 $\mathrm{cm}^{-1}$ belongs to the trans conformer (calculated spectrum 3), while the peaks at 2140 and $2227 \mathrm{~cm}^{-1}$ (calculated spectrum 2) belong to the cis conformer of $2^{\bullet+}$.

Calculations. In order to understand and explain the apparently spontaneous ring-opening of the radical cation of cyclobutenedione, we explored the potential surfaces for this process and for the interconversion of the two conformers of the bisketene radical cation. The results of these efforts are depicted in Figure 3, which shows that, indeed, there is almost no barrier for the decay of $\mathbf{1}^{\bullet+}$, for two reasons: first the reaction, which had been only slightly exothermic in neutral $\mathbf{1}$, is now exothermic by $21.7 \mathrm{kcal} / \mathrm{mol}$ (on a G4 enthalpy scale), and second, the electron that is removed on ionization comes from the HOMO which is $\sigma$-bonding along the $\mathrm{C}-\mathrm{C}$ bond that links the two carbonyl groups in $\mathbf{1}$, which leads to a substantial weakening of that bond. This expresses itself for example in the fact that, at the equilibrium structure of $\mathbf{1}^{\bullet+}$, that $\mathrm{C}-\mathrm{C}$ bond has a length of $2.1 \AA$.
However, to decay to $c i s-2^{\bullet+}$ the system must pass from the ${ }^{2} \mathrm{~A}_{1}$ surface of ground-state $\mathbf{1}^{\bullet+}$ to the ${ }^{2} \mathrm{~A}_{2}$ surface of cis- $\mathbf{2}^{\bullet+}$, and to do that, the symmetry must be lowered to $C_{2}$ (where both states become $\left.{ }^{2} \mathrm{~A}\right)$. This is indeed what is found at the transition state (TS in Figure 3) which lies, however, only 0.57 $\mathrm{kcal} / \mathrm{mol}$ above $\mathbf{1}^{\bullet+}$, on a G4 enthalpy scale, which explains why the decay to $2^{\bullet+}$ is spontaneous.

From the IR spectra in Figure 1 we concluded that we see both stable conformations of $2^{\bullet+}$, so the question poses itself how trans- $2^{\bullet+}$ can be reached. From the right side of Figure 3 we see that the interconversion of the two conformers of $2^{\bullet+}$ involves a state crossing, even in $C_{2}$ symmetry; i.e., at $90^{\circ}$ there is a conical intersection (CI) between the ${ }^{2} \mathrm{~A}$ and the ${ }^{2} \mathrm{~B}$ surfaces.

Ground-state reactions always circumvent conical intersections; their transition states lie in the "moat" that surrounds them. The only way for $2^{\bullet+}$ to pass from the ${ }^{2} \mathrm{~A}$ to the ${ }^{2} \mathrm{~B}$ surface is by localizing the SOMO, and hence the spin, in onehalf of the molecule (which leads to a total loss of symmetry), so the coefficients in the other half can re-emerge with the opposite sign (see MOs near the CI in Figure 3).

Unfortunately, as we have explained before, ${ }^{8}$ such transformations cannot be followed by any quantum chemical method that accounts for dynamic electron correlation (which cannot be ignored when one compares localized and delocalized species!). Therefore, we are unable to provide a reliable prediction for the activation energy for the cis-trans interconversion of $2^{\bullet+}$, and we must content ourselves with an estimate of around $10 \mathrm{kcal} / \mathrm{mol}$.

The question remains how trans- $\mathbf{2}^{\bullet+}$ is formed from $\mathbf{1}^{\bullet+}$. In experiments where Ar-matrices are exposed to X-irradiation, the ionization of added substrates occurs by charge transfer from $\mathrm{Ar}^{\bullet+}$ (or, more precisely, $\mathrm{Ar}_{n}^{\bullet+}$, because charge or spin are not localized on single Ar atoms in an Ar crystal), a process which is invariably quite exothermic. As $\mathrm{Ar}$ is a very poor heat conductor, the excess energy that is thus imparted on the incipient substrate cations is only hesitatingly dissipated, and is therefore available to surmount small activation barriers. The spontaneous ring-opening of $\mathbf{1}^{\bullet+}$ endows nascent $2^{\bullet+}$ with an 
additional $\mathrm{eV}$ of excess energy, and perhaps the two combined effects suffice to make it possible for a part of cis- $\mathbf{2}^{\bullet+}$ to cross over to trans $-2^{\bullet+}$. However, we cannot exclude that trans- $2^{\bullet+}$ arises by a different mechanism, directly from $\mathbf{1}^{\mathbf{}}$, as in the case of the radical cation of cyclobutene which undergoes a concerted ring-opening to yield the radical cation of transbutadiene.

\section{CONCLUSION}

We have generated the radical cation of cyclobutenedione, $\mathbf{1}^{\bullet+}$, by X-irradiation of an Ar matrix containing 1, and observed its spontaneous decay to the radical cation of bisketene $2^{\bullet+}$ (buta1,3-diene-1,4-dione) which is in large part reneutralized to 2 by the electrons liberated by the X-irradiation of Ar. This allowed us to characterize for the first time parent 2 and its radical cation by matrix IR spectroscopy. The spectra, and the accompanying quantum chemical calculations, lead us to the conclusion that neutral 2 exists only in the form of a single conformer where the two ketene moieties are nearly perpendicular, whereas $\mathbf{2}^{\bullet+}$ has two stable planar conformers, both of which are formed on ionization of $\mathbf{2}$. According to highlevel calculations the decay of $\mathbf{1}^{\bullet+}$ to $\mathbf{2}^{\bullet+}$ is virtually activationless, whereas the interconversion of the two conformers of $2^{\bullet+}$ involves a barrier that cannot be precisely calculated but is probably in the vicinity of $10 \mathrm{kcal} / \mathrm{mol}$, because it involves a state crossing.

\section{METHODS}

Cyclobutenedione $\mathbf{1}$ was synthesized according to the procedure described in ref $2 \mathrm{f}$.

For the matrix isolation experiments, 1 was mixed in a 1:1000 ratio with a 9:1 mixture of $\mathrm{Ar}$ and $\mathrm{N}_{2}$ (where the latter is added to improve the optical quality of the matrices) in a $2000 \mathrm{~mL}$ storage vessel to a total pressure of $100 \mathrm{mbar}$, about half of which was deposited over ca. $1 \mathrm{~h}$ on a CsI window held at $20 \mathrm{~K}$ inside the cold head of a closedcycle cryostat. After the deposition was complete, the heater of the cryostat was switched off, so the temperature of the matrix descended to $10 \mathrm{~K}$. Photolysis was done with a low-pressure $\mathrm{Hg}$ lamp $(254 \mathrm{~nm})$ or a medium-pressure $\mathrm{Hg} / \mathrm{Xe}$ lamp fitted with an interference filter $(365 \mathrm{~nm})$ or a cutoff filter $(>515 \mathrm{~nm})$. X-irradiation was effected by means of a source tube containing a tungsten anode that was operated at $40 \mathrm{kV} / 40 \mathrm{~mA}$, which gives off Bremsstrahlung that under these conditions peaks at ca. $0.5 \AA / 2.5 \mathrm{keV}$. Under the impact of this radiation, electron-hole pairs form in the Ar matrix, a few of which separate and diffuse (in the form of polarons) through the solid until they are trapped by added substrates or impurities with a lower oxidation or reduction potential, respectively, than Ar.

Initially, we added an equivalent of methylene chloride to serve as electron scavenger, ${ }^{4}$ but it turned out that this did not make any difference so we assume that the electrons are scavenged either by 1 or by some impurities such as $\mathrm{CO}_{2}$ (however, we found no evidence for the formation of $\mathbf{1}^{\bullet-}$ or $2^{\bullet-}$ ). As the radical cations that are formed by hole trapping accumulate, they begin to contribute increasingly to the scavenging of electrons, until a steady state is attained (after ca. $90 \mathrm{~min}$ under our experimental conditions) where holes and electrons are scavenged at the same rate.

If the primary radical cations are chemically stable, longer $\mathrm{X}$ irradiation leads to no further spectral changes, except for the slow formation of side products. If, however, the primary radical cations undergo fragmentation or rearrangement, the electrons will be scavenged by the secondary cations to yield rearranged or fragmented neutral species, as it is the case in the example shown in this paper. On continuing X-irradiation those species continue to accumulate past the point where the cation production has leveled off, and they may thus become much more prominent than the primary cations, as in the present case.
In the quantum chemical calculations, all structures (minima and transition states) were first optimized by the standard B3LYP/6-31G* method which also served to compute vibrational frequencies (which were scaled by 0.968 for neutrals and 0.960 for radical cations for comparison with experiment) and IR intensities. Subsequently, the structures were reoptimized and their energies were computed according to the G4 composite protocol ${ }^{10}$ which is expected to yield heats of formation to an accuracy of $0.65 \mathrm{kcal} / \mathrm{mol}$, and probably even more accurate relative energies. All calculations were done with the Gaussian program suite, ${ }^{11}$ the B3LYP and G4 energies are given in the Supporting Information.

An interesting problem occurred in the case of the $C_{2 h}$ structure of 2 that resides in a shallow minimum at the B3LYP level (which is also used to optimize structure within the G4 protocol, albeit with a much larger basis set), but where the transition state for its decay to the orthogonal equilibrium structure lies ca. $1 \mathrm{kcal} / \mathrm{mol}$ below the $C_{2 h}$ structure at the (empirically corrected) $\operatorname{CCSD}(\mathrm{T})$ level that is used in G4. This shows that such composite protocols should not be used blindly but with a measure of circumspection.

\section{ASSOCIATED CONTENT}

\section{Supporting Information}

Complete ref 11; results of B3LYP and G4 calculations for 1, 2, and their radical cations, including Cartesian coordinates for all stationary points.

\section{AUTHOR INFORMATION}

\section{Corresponding Author}

*E-mail: thomas.bally@unifr.ch.

\section{Notes}

The authors declare no competing financial interest.

\section{ACKNOWLEDGMENTS}

This work was carried out as part of project no. 200020-121747 of the Swiss National Science Foundation.

\section{REFERENCES}

(1) (a) Allen, A. D.; Ma, J.; McAllister, M. A.; Tidwell, T. T.; Zhao, D.-C. Acc. Chem. Res. 1995, 28, 265-271. (b) Tidwell, T. T. Ketenes, 2nd ed.; Wiley-Interscience: Hoboken, 2006. (c) Tidwell, T. T. Eur. J. Org. Chem. 2006, 563-576. (d) Allen, A. D.; Godoy, J.; Fu, N.; Nagy, M.; Spadaro, S.; Tidwell, T. T; Vukovic, S. J. Am. Chem. Soc. 2008, 130, 2386-2387. (e) Bock, H.; Ried, W.; Stein, U. Chem. Ber. 1981, 114, 673-683. (f) Mallory, F. B.; Roberts, J. D. J. Am. Chem. Soc. 1961, 83, 393-397. (g) Dejmek, M. M.; Selke, R. Angew. Chem., Int. Ed. 1998, 37, 1540-1542. (h) Dejmek, M. M.; Selke, R. Synlett 2000, 1321. (i) Colomvakos, J. D.; Egle, I.; Ma, J.; Pole, D. L.; Tidwell, T. T.; Warkentin, J. D. J. Org. Chem. 1996, 61, 9522-9527.

(2) (a) Maier, G.; Reisenauer, H. P.; Sayrac, T. Chem. Ber. 1982, 115 , 2192-2201. (b) Breda, S.; Reva, I.; Lapinski, L.; Fausto, R. J. Phys. Chem. A 2006, 110, 11034-11045. (c) Mosandl, T.; Wentrup, C. J. Org. Chem. 1993, 58, 747-749. (d) Allen, A. D.; Colomvakos, J. D.; Diederich, F.; Egle, I.; Hao, X.; Liu, R.; Lusztyk, J.; Ma, J.; McAllister, M. A.; Rubin, Y.; Sung, K.; Tidwell, T. T.; Wagner, B. D. J. Am. Chem. Soc. 1997, 119, 12125-12130. (e) Egle, I.; Lai, W.-Y.; Moore, P. A.; Renton, P.; Tidwell, T. T.; Zhao, D.-c. J. Org. Chem. 1997, 62, 18-25. (f) Allen, A. D.; Tidwell, T. T. Can. J. Chem. 1999, 77, 802-805. (g) Allen, A. D.; Moore, P. A.; Missiha, S.; Tidwell, T. T. J. Org. Chem. 1999, 64, 4690-4696. (h) Jewell, C. F., Jr.; Liebeskind, L.; Williamson, M. J. Am. Chem. Soc. 1985, 107, 6715-6716. (i) Miller, R. D.; Kirchmeyer, S. J. Org. Chem. 1993, 58, 90-94. (j) Miller, R. D.; Theis, W.; Heilig, G.; Kirchmeyer, S. J. Org. Chem. 1991, 56, 1453-1463. (k) Tomioka, K.; Yamamoto, K. J. Chem. Soc., Chem. Commun. 1995, 1961-1962. (1) Diederich, F.; Rubin, Y.; Chapman, O. L.; Goroff, N. S. Helv. Chim. Acta 1994, 77, 1441-1457.

(3) Bally, T.; Michalak, J. J. Photochem. Photobiol. A. 1992, 60, 185. 
(4) Bally, T. In Radical Ionic Systems; Lund, A., Shiotani, M., Eds.; Kluwer: Dordrecht, 1991.

(5) In butadiene the twisted geometry lies ca $6 \mathrm{kcal} / \mathrm{mol}$ higher than the planar trans structure: Pratt, L. R.; Hsu, C. S.; Chandler, D. J. Chem. Phys. 1978, 68, 4202.

(6) Brown, F. R.; Finseth, D. H.; Miller, F. A.; Rhee, K. H. J. Am. Chem. Soc. 1975, 97, 1011.

(7) A reviewer has suggested that these bands may be due to reaction of the ketenecarbene $\mathrm{O}=\mathrm{C}=\mathrm{CH}-\ddot{\mathrm{C}}-\mathrm{H}$ that arises upon loss of $\mathrm{CO}$ from 2 (or its radical cation) with the $10 \% \mathrm{~N}_{2}$ that we add to our matrices to improve their optical quality to form diazopropenone $(\mathrm{O}=\mathrm{C}=\mathrm{CH}-\mathrm{CH}=\mathrm{N}=\mathrm{N})$, which may have bands in the 2100-200 $\mathrm{cm}^{-1}$ region. Unfortunately, we have no way to confirm or reject this interesting hypothesis which we consider, however, improbable for various reasons.

(8) (a) Müller, B.; Bally, T.; Gerson, F.; de Meijere, A.; von Seebach, M. J. Am. Chem. Soc. 2003, 125, 13776. (b)

(9) Sastry, G. N.; Bally, T.; Hrouda, V.; Cársky, P. J. Am. Chem. Soc. 1998, 120, 9323.

(10) Curtiss, L. A.; Redfern, P. C.; Raghavachari, K. J. Chem. Phys. 2007, 126, 084108.

(11) Frisch, M. J. et al. Gaussian 09, Revision A.02; Gaussian, Inc.: Wallingford, CT, 2003 (for the complete reference, see the Supporting Information). 